\title{
The Impacts of Structure Position and Size Change on Ultrasonic Transmission Properties
}

\author{
Jialing $\mathrm{Hu}^{1}$, a , Lei $\mathrm{Li}^{1, \mathrm{~b}}$, Aiming $\mathrm{Ji}^{1, \mathrm{c}}$, Li Sun ${ }^{1, \mathrm{~d}}$, Canyan Zhu ${ }^{1, \mathrm{e}}$, Lijun Zhang ${ }^{1, \mathrm{f}}$ \\ and Ling-Feng Mao, $\mathrm{g}$ \\ ${ }^{1}$ Institute of Intelligent Structure and System, Soochow University, Suzhou, 215006, P. R. China \\ a20124246010@suda.edu.cn, ${ }^{b}$ lei_li56@163.com, jijiaiming@suda.edu.cn, 'sunli901012@163.com,

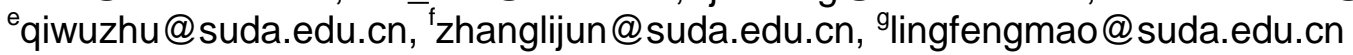

Keywords: structure position, structure size, ultrasonic detection, FDTD simulation

\begin{abstract}
Based on the finite differential time-domain (FDTD) method, two types of aluminum plate samples with one and two circular cavities are established. The ultrasonic transmission signal and acoustic pressure distribution for samples with different cavity positions $(x)$ and diameters $(d)$ are obtained through FDTD simulations. The results show that when $x$ is between 6 wavelengths $(15 \mathrm{~mm})$ and 14 wavelengths $(35 \mathrm{~mm})$ of the ultrasound, the power of the transmitted ultrasonic signal increases dramatically for the two cavity diameters of $d=2 \lambda$ and $d=\lambda$. Such an increase is especially large when the cavity is in the middle and the maximum relative change is about $260 \%$ when $d=2 \lambda$. However, the power does not change much with changing $\mathrm{x}$ and is close to the sample with no cavity when $d=0.5 \lambda$. It also shows that the transmitted power is weakened for the sample with two cavities due to the coupling effect between them. These results may have potential use in ultrasonic detection.
\end{abstract}

\section{Introduction}

Ultrasonic detection technology (UDT) is one of the most frequently used non-destructive testing methods. It is widely used in defect recognition and detection [1]. Defect parameters that are essential to the quality of ultrasonic detection are defect position in the object and size. A number of investigations address on them [2]. When conducting ultrasonic detection, the sample size may affect the detection results [3], which is similar to the quantum size effects in nano-electronics [4]. Thus, necessary modifications should be made to the detection results when sample width is a few wavelengths. Also, the structure position and size may have an impact on ultrasonic properties, which should be taken into account when making these modifications. UDT result is correlative with ultrasonic transmission signal and sound field, and numerical simulation can effectively analyze ultrasonic sound field characteristics [5]. Finite differential time-domain (FDTD) method is a good way to conduct ultrasonic simulation [6]. This paper establishes two types of aluminum plate samples and studies the acoustic field characteristics of the samples through FDTD method.

\section{Method}

Simulation Tool. All the simulation results are performed by using SimSonic2D, which is a 3rd party software suite for the simulation of ultrasound propagation, based on finite-difference time-domain (FDTD) computations of the elastodynamic equations [7],

$$
\begin{gathered}
\rho(\mathbf{x}) \frac{\partial v_{i}}{\partial t}(\mathbf{x}, t)=\sum_{j=1}^{d} \frac{\partial T_{i j}}{\partial x_{j}}(\mathbf{x}, t)+f_{i}(\mathbf{x}, t) \\
\frac{\partial T_{i j}}{\partial t}(\mathbf{x}, t)=\sum_{j=1}^{d} \sum_{i=1}^{d} c_{i j k l}(\mathbf{x}) \frac{\partial v_{k}}{\partial x_{l}}(\mathbf{x}, t)+\theta_{i j}(\mathbf{x}, t)
\end{gathered}
$$

Here $x$ and $t$ are the space and time variables. $\rho(\mathbf{x})$ is the mass density and $c(\mathbf{x})$ is the fourth rigidity tensor. These parameters entirely define the materials properties. 
In simulations, the amount of computer resource taken by calculations will increase sharply when the dimensions of the simulation model increase. So we consider the two-dimensional model. The simulation model is a thin aluminum plate with circular cavities. Perfectly matched layers (PMLs) [8] are defined against to the simulation boundaries which ensured non-reflective propagation of wave incident on them. Acoustic absorption and dispersion of the material are not considered in the simulations.

Simulation Model. The parameters are set as follows: the length and width of the aluminum plate is $50 \mathrm{~mm}$ and $7.5 \mathrm{~mm}$, respectively. In the FDTD simulations, spatial grid step in $x$ and $y$ dimensions are both $0.01 \mathrm{~mm}$. The time step is $5.53 \mathrm{~ns}$ to ensure calculation precision. The geometry of the source object is a line array. The length of the source array is $5 \mathrm{~mm}$. The incident wave is in the form of normal incidence. The source signal is the continuous sinusoidal ultrasonic signal in the form of,

$$
s(t)=\sin \left(2 \pi f_{0} t\right) .
$$

Here $\mathrm{f}_{0}$ is the frequency of the ultrasonic signal, and it is chosen as $2.53 \mathrm{MHz}$ in the simulation. The receiver is placed on the right side centre of the sample, and it consists of one grid point. Adopted material parameters are $\rho_{\text {aluminum }}=2700 \mathrm{~kg} / \mathrm{m}^{3}, \rho_{\text {air }}=1.21 \mathrm{~kg} / \mathrm{m}^{3}$ for densities and $c_{\text {aluminum }}=6325$ $\mathrm{m} / \mathrm{s}, c_{\text {air }}=334.5 \mathrm{~m} / \mathrm{s}$ for longitudinal speeds of sound in the homogeneous media, respectively [9]. Thus the wavelength of the ultrasound $\lambda$ in aluminum is $2.5 \mathrm{~mm}$. To investigate the influence of structure position and size change on ultrasonic transmission, two simulation models are established which are illustrated in Fig.1. In Fig.1a, sample 1 is shown with one circular cavity. The distance of its center to the left side $x$ and the diameter $d$ are varied. In Fig.1b, sample 2 is shown with two cavities and the distance between them is $a$.

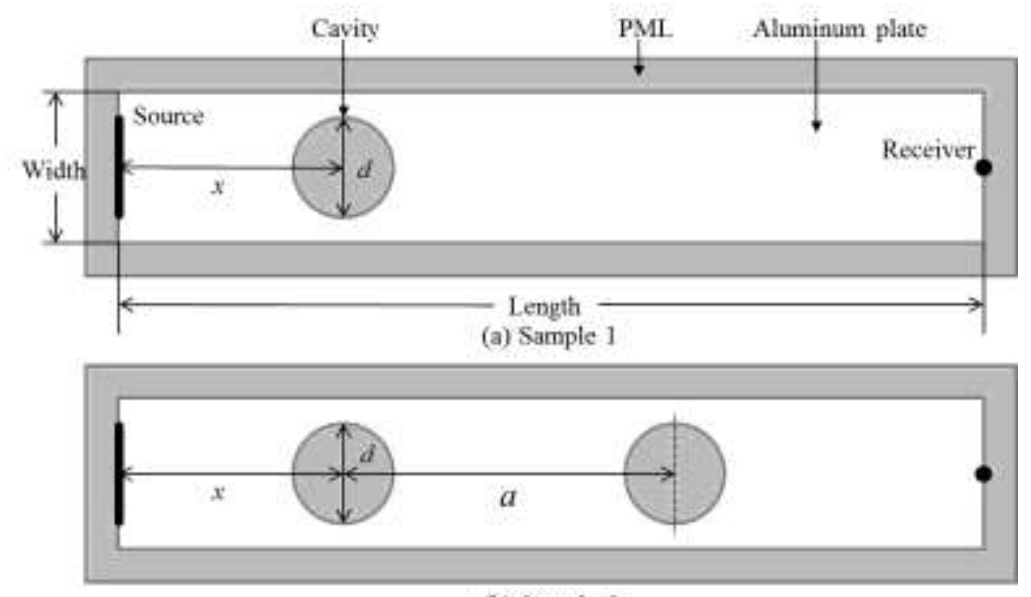

(b) Sample 2

Fig. 1. Diagrammatic sketch of the samples considered.

\section{Numerical Results and Discussion}

Change of Structure Position. The transmitted ultrasonic signal in time-domain obtained by the receiver and the snapshots of acoustic field at $80 \mu \mathrm{s}$ with different cavity position $x$ for sample 1 when $d=2 \lambda(5 \mathrm{~mm})$ are depicted in Fig.2a-b, respectively. It can be seen from Fig.2a that the change of $x$ can significantly influence the amplitude of the transmitted ultrasonic signal. The normalized steady-state signal amplitude for sample without the cavity is chosen as the reference. The signal amplitude decreases dramatically when $x$ is $10 \mathrm{~mm}$ compared to the sample with no cavity. The signal amplitude reaches the highest when $x$ is $25 \mathrm{~mm}$ (cavity in the middle), and decreases again when $x$ is $40 \mathrm{~mm}$.

The distribution of acoustic pressure at $80 \mu \mathrm{s}$ can be seen from Fig.2b. For the sample with no cavity, the acoustic distribution is relatively symmetrical. For the samples with one cavity, reflection and scattering appear when the ultrasonic wave transmits to the cavity. It can be seen from the figure that the change of $x$ has an impact on the acoustic pressure distribution. When $x$ is 
$25 \mathrm{~mm}$, obvious scattered wave can be seen around the cavity and the transmitted acoustic pressure is very large. In addition, the interference of the incident and reflected wave from the cavity is strong, which makes the acoustic pressure on the left side of the cavity large. As for the sample when $x$ is $10 \mathrm{~mm}$, the acoustic pressure is strong on the left side of the cavity but it is significantly smaller on the right side which indicates the transmitted acoustic pressure decreases dramatically. When $x$ is $40 \mathrm{~mm}$, the scattered wave around the cavity is relatively small and the reflected and transmitted acoustic pressure both decreases compared to the sample when $x$ is $25 \mathrm{~mm}$.

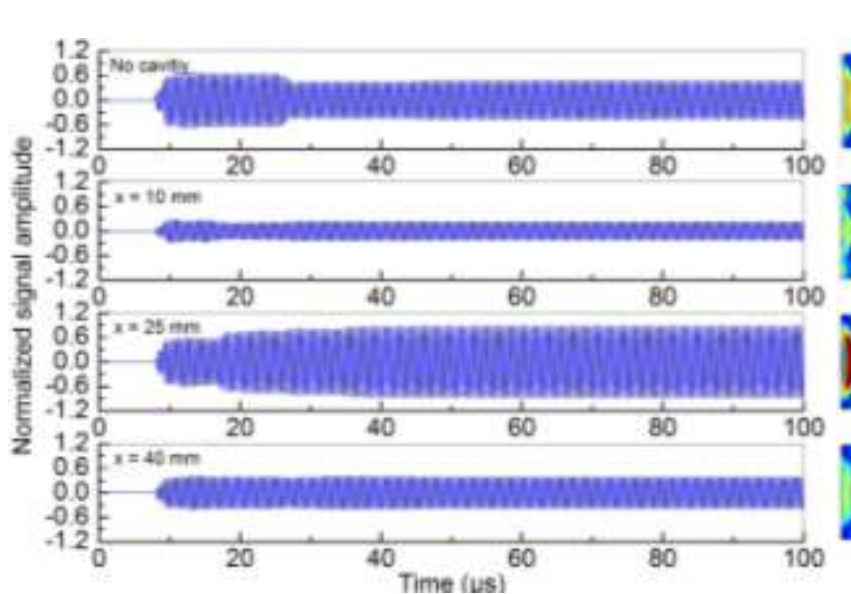

(a)

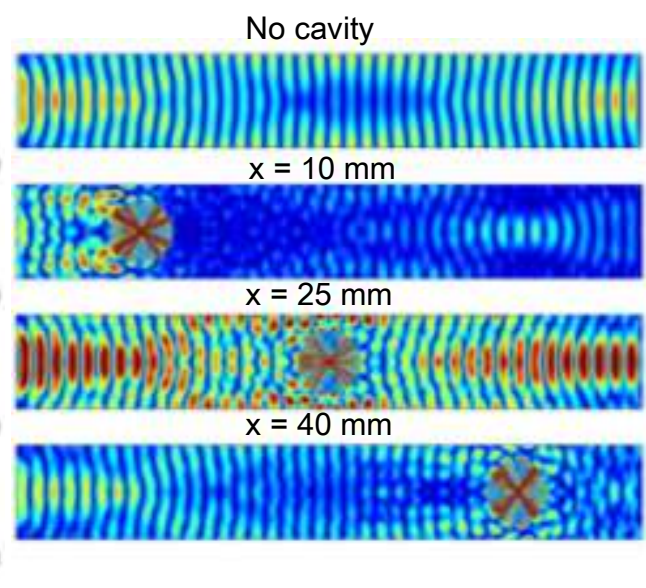

(b)

Fig. 2. Results for sample 1 when $d=2 \lambda$ : (a) Transmitted ultrasonic signal in time-domain, (b) Snapshots of acoustic field distribution at $80 \mu \mathrm{s}$

Fig. 3 gives the mean steady-state power (circular symbol) and its relative change (square symbol) as a function of $x$ for sample 1 when $d=2 \lambda$. The reference (dash line) indicates the result for the sample with no cavity. The power increases as $x$ increases and reaches the peak when $x$ is 30 $\mathrm{mm}$. Then, the power decreases as $x$ increases when $x$ is larger than $30 \mathrm{~mm}$. Checking this figure in detail, we can see that the power is above the reference when $x$ is larger than $15 \mathrm{~mm}$ ( 6 wavelengths) and smaller than $35 \mathrm{~mm}$ (14 wavelengths) which means scattering is strong when the cavity is located within these areas.

Assuming the mean steady-state power for the sample with no cavity is $p_{0}$, and the mean steady-state power is $p$. Then its relative change $r$ can be defined in the form of,

$$
r=\frac{\left|p-p_{0}\right|}{p_{0}} .
$$

Fig. 3 shows that the largest relative change of mean steady-state power of the transmitted ultrasound is $260 \%$ when $\mathrm{x}$ is $30 \mathrm{~mm}$.

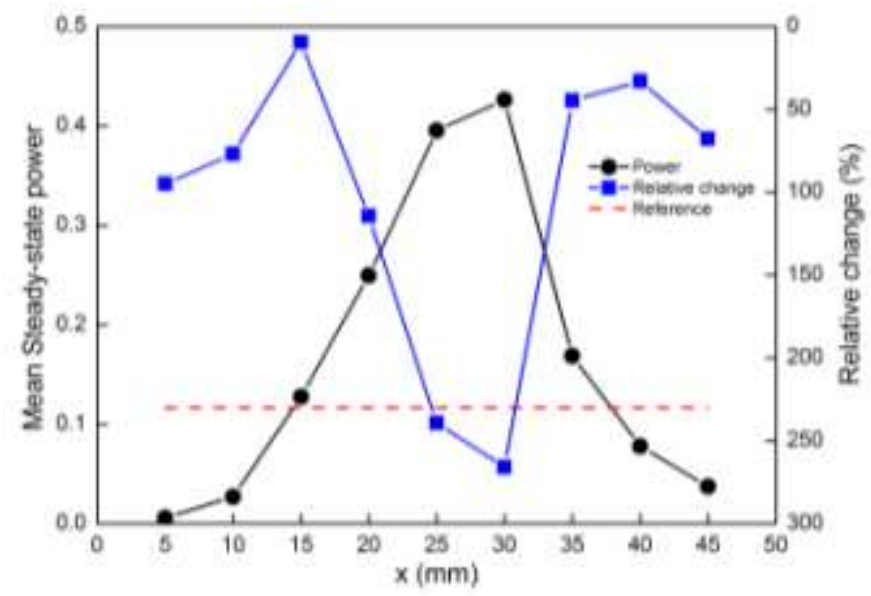

Fig. 3. Mean steady-state power and its relative change as a function of $x$ for sample 1 when $d=2 \lambda$

(Reference is the result for sample with no cavity) 
In order to investigate the influence of the adding of cavity on ultrasonic transmission properties, we fix one cavity at $x=15 \mathrm{~mm}$, which is the starting position of scattering, and add one cavity at the right side of the fixed cavity. Then, we study the results with changing $a$ (distance between the two cavities).

The transmitted ultrasonic signal in time-domain obtained by the receiver and the snapshots of acoustic field at $80 \mu \mathrm{s}$ are depicted in Fig.4a-b, respectively. From Fig.4a, we can see that the increase of $a$ can decrease the amplitude of the transmitted ultrasonic signal compared to the sample without the adding cavity. The distribution of acoustic pressure at $80 \mu \mathrm{s}$ can be seen from Fig. $4 \mathrm{~b}$. Obvious scattered wave can also be seen around the cavity and the reflected acoustic pressure is large for different $a$. However, the transmitted acoustic pressure decreases with increasing $a$.

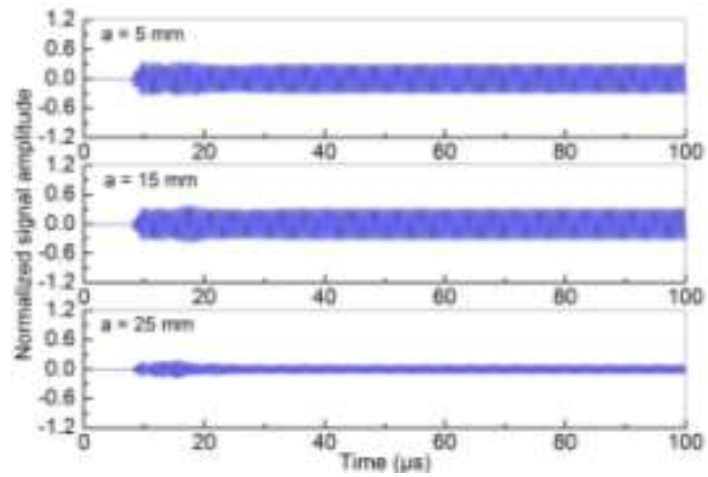

(a)

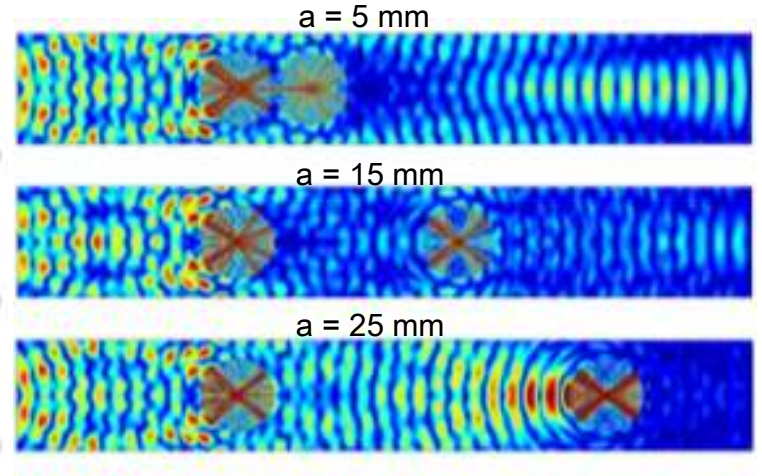

(b)

Fig. 4. Results for sample 2 when $d=2 \lambda$ : (a) Transmitted ultrasonic signal in time-domain, (b) Snapshots of acoustic field distribution at $80 \mu \mathrm{s}$ (the distance from the center of the left cavity to the left side is $15 \mathrm{~mm}$ )

Fig. 5 gives the mean steady-state power (circular symbol) and its relative change (square symbol) as a function of $a$ when $d=2 \lambda$. The reference (dash line) indicates the result for the sample without the adding cavity, that is, sample 1 when $x=15 \mathrm{~mm}$. It can be clearly seen that the power is much smaller than the reference and the largest relative change is about $100 \%$. This is because coupling effect exists between cavities which weakens the transmission of ultrasound.

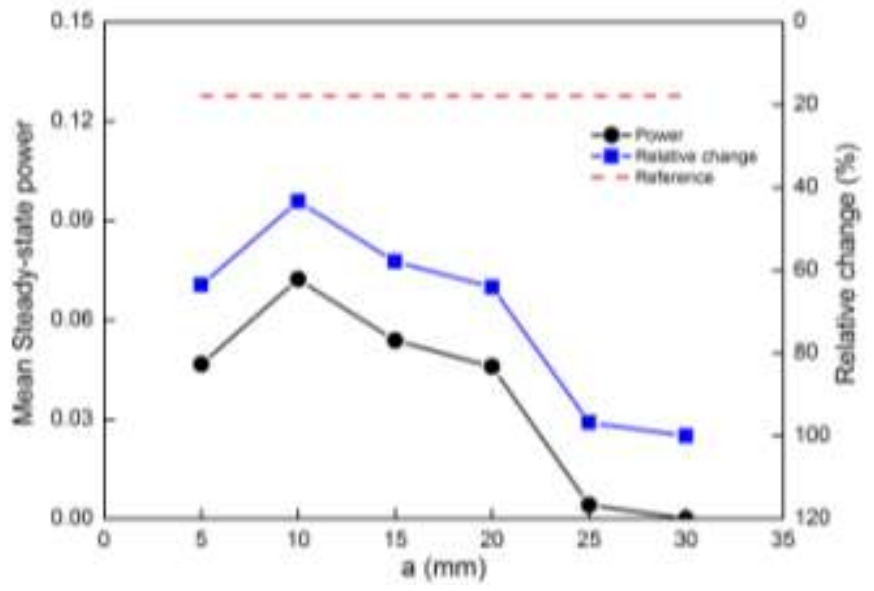

Fig. 5. Mean steady-state power and its relative change as a function of $a$ for sample 2 when $d=2 \lambda$

(Reference is the result for sample with one cavity when $x=15 \mathrm{~mm}$ )

Change of Structure Position. In order to study the impact of structure position on ultrasonic transmission under different structure sizes, the diameter of the cavity is changed for sample 1 . The transmitted ultrasonic signal in time-domain obtained by the receiver and the snapshots of acoustic field at $80 \mu s$ when $d=\lambda$ and $d=0.5 \lambda$ are depicted in Fig.6a-b, and Fig.7a-b, respectively. For the sample when $d=\lambda$, the change of $x$ still affect the transmitted ultrasonic signal amplitude obviously, which can be seen from Fig.6a. The reflected and transmitted acoustic pressure also changes greatly with changing $x$ and strong scattering occurs when $x=25 \mathrm{~mm}$ (cavity in the middle), as can be seen in Fig.6b. 


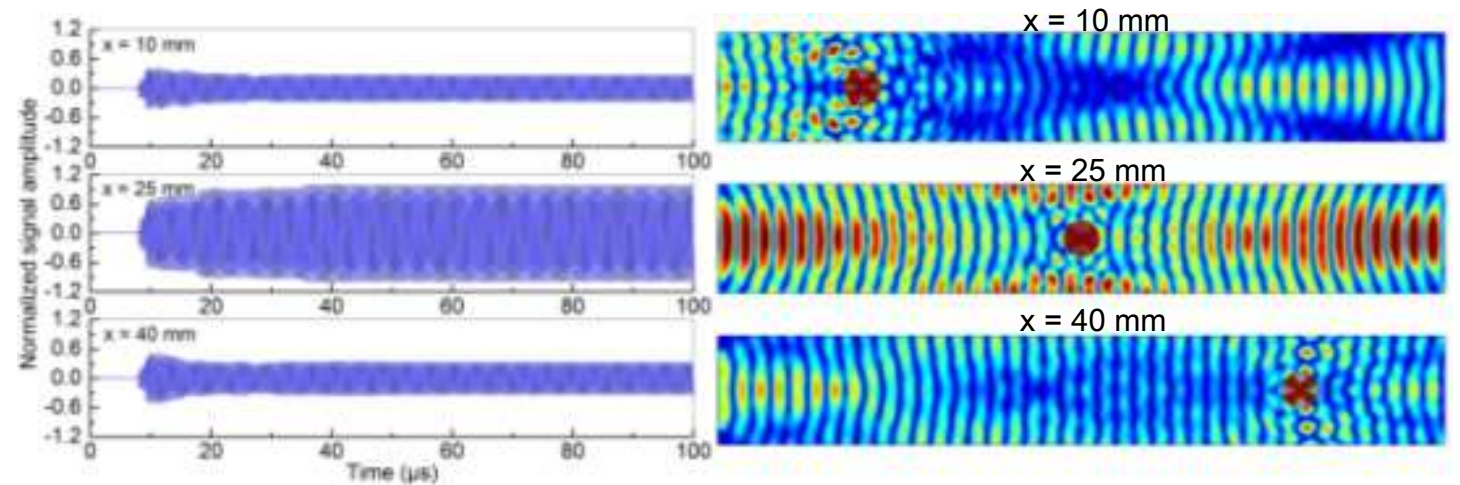

(a)

(b)

Fig. 6. Results for sample 1 when $d=\lambda$ : (a) Transmitted ultrasonic signal in time-domain, (b)

Snapshots of acoustic field distribution at $80 \mu \mathrm{s}$

However, for the sample when $d=0.5 \lambda$, the change of the transmitted ultrasonic signal amplitude with changing $x$ is not as great as the former two diameters, which can be seen from Fig.7a. So is the reflected and transmitted acoustic pressure, as can be seen in Fig. $7 \mathrm{~b}$.

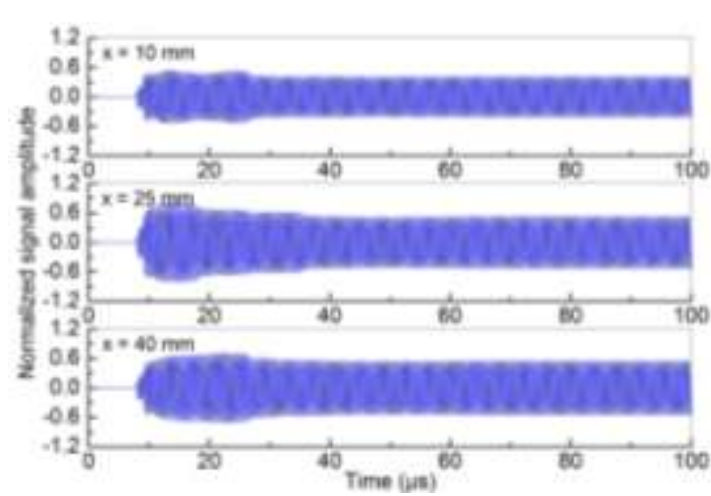

(a)

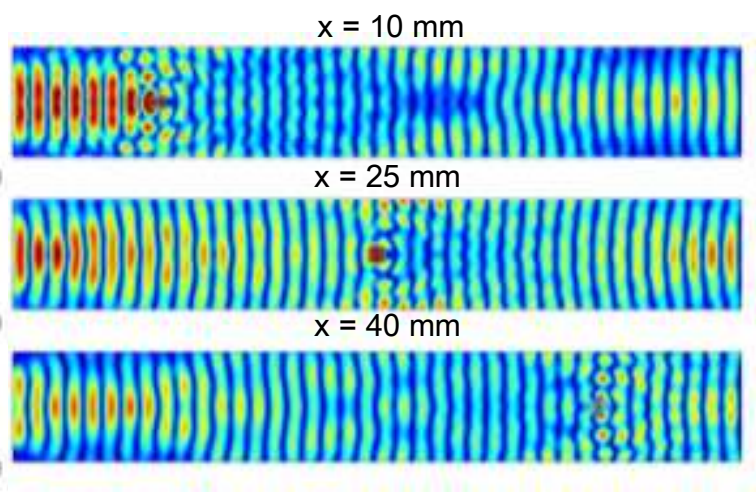

(b)

Fig. 7. Results for sample 1 when $d=0.5 \lambda$ : (a) Transmitted ultrasonic signal in time-domain, (b)

Snapshots of acoustic field distribution at $80 \mu \mathrm{s}$

Fig. 8 depicts the mean steady-state power as a function of $x$ for sample 1 when $d=2 \lambda, \lambda$ and $2 \lambda$. The reference (dash line) indicates the result for the sample with no cavity. For the two samples when $d=2 \lambda$ and $d=\lambda$, the power increases with increasing $x$ and reaches the peak when $x$ is $25 \mathrm{~mm}$ and $30 \mathrm{~mm}$, respectively. Then, the power decreases as $\mathrm{x}$ increases when $\mathrm{x}$ is larger. In addition, the power is above the reference when $\mathrm{x}$ is larger than $15 \mathrm{~mm}$ ( 6 wavelengths) and smaller than $35 \mathrm{~mm}$ (14 wavelengths) for both samples. However, for the sample when $d=0.5 \lambda$, the power is close to the reference and the oscillation is small when $\mathrm{x}$ is changing.

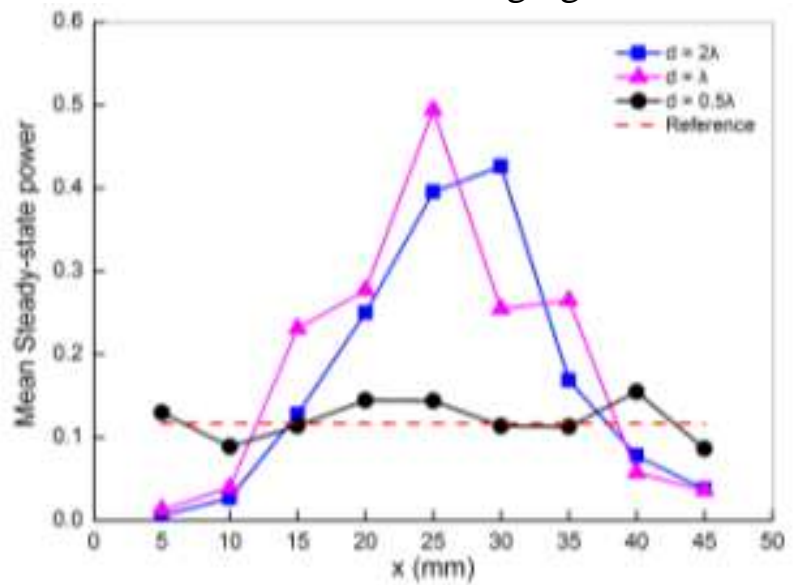

Fig. 8. Mean steady-state power and its relative change as a function of $x$ for sample 1 when $d=2 \lambda$, $d=\lambda$ and $d=0.5 \lambda$ (Reference is the result for sample with no cavity) 


\section{Conclusion}

In conclusion, the impact of structure position and size change on acoustic filed properties has been numerically investigated by FDTD method. Two aluminum samples with one and two circular cavities are considered in the simulation. Simulation results show that for the sample with one cavity, when $d=2 \lambda$ and $d=\lambda$, the power of the transmitted ultrasonic signal increases greatly when $x$ is between 6 wavelengths $(15 \mathrm{~mm})$ and 14 wavelengths $(35 \mathrm{~mm})$ of the ultrasound. Such an increase is especially large when the cavity is located in the middle of the tested sample. The largest relative change of mean steady-state power compared to the sample with no cavity is about $260 \%$ when $d=2 \lambda$. However, for the sample when $d=0.5 \lambda$, the power is close to the reference with changing $x$. As for the sample with two cavities, the transmitted ultrasonic power decreases compared to the sample with one cavity and its largest relative change is about $100 \%$. This is because coupling effect exists between cavities. Hence, the adding of the cavity decreases the transmitted ultrasonic power. These results imply the structure location and size change can affect the ultrasonic transmission properties, which are expected to have applications in ultrasonic detection.

\section{References}

[1] C. Fan, M. Pan, F. Luo and B.W. Drinkwater, Multi-frequency time-reversal-based imaging for ultrasonic nondestructive evaluation using full matrix capture, IEEE. Trans. Ultrason. Ferroelectr. Freq. Control. 61 (2014) 2067-2074.

[2] G.De Angelis, M. Meo, D.P. Almond, S.G. Pickering, S.L. Angioni. A new technique to detect defect size and depth in composite structures using digital shearography and unconstrained optimization. NDT \& E. Int. 45 (2012) 91-96.

[3] J.C. Piquette, Technique for detecting the presence of finite sample-size effects in transmitted-wave measurements made on multilayer underwater acoustic panels, J. Acoust. Soc. Am. 90 (1991) 2831.

[4] L.F. Mao, A theoretical analysis of field emission from graphene nanoribbons, Carbon. 49 (2011) 2709-2714.

[5] Z.G. Zhou and D. Wei, Analysis of Ultrasonic Sound Field Characteristic with FDTD, Chin. J. Mech. Eng. 46 (2010) 9-13.

[6] Y. Nagatani, K. Mizuno, T. Saeki, M. Matsukawa, T. Sakaguchi and H. Hosoi, Numerical and experimental study on the wave attenuation in bone-FDTD simulation of ultrasound propagation in cancellous bone, Ultrasonics. 48 (2008) 607-612.

[7] E. Bossy, M. Talmant and P. Laugier, Three-dimensional simulations of ultrasonic axial transmission velocity measurement on cortical bone models, J. Acoust. Soc. Am. 115 (2004) 2314-2324.

[8] F.D. Hastings, J.B. Schneider and S.L. Broschat, Application of the perfectly matched layer (PML) absorbing boundary condition to elastic wave propagation, J. Acoust. Soc. Am. 100 (1996) 3061-3069.

[9] O.A. Kaya, A. Cicek and B. Ulug, Focusing with two-dimensional angular-symmetric circular acoustic lenses. Acoust. Phys. 57 (2011) 292-300. 